\title{
Anticuerpos antifosfolípidos en nefropatía membranosa idiopática
}

\author{
Leopoldo Ardiles A, Eileen Blackburn T, Paula Alruiz Sa, \\ Claudio Flores W, Sergio Mezzano A.
}

\author{
Antiphospholipid antibodies in \\ idiopathic membranous nephropathy
}

Background: Antiphospholipid antibodies have been found in the sera from patients with idiopathic and secondary glomerulopathies, mainly related to lupus. No special attention has been devoted to idiopathic membranous nephropathy, a glomerular disease with a high frequency of thrombotic complications, particularly of the renal vein. Aim: To study the presence and significance of antiphospholipid antibodies in idiopathic membranous nephropathy. Material and methods: Anticardiolipin and anti- $\aleph_{2}$-glycoproteinI IgG antibodies were measured in serum samples from 21 patients with idiopathic membranous nephropathy (age range 11-75 years, 5 female). The medical records of 20 of these patients were reviewed, looking for vascular complications and nephrological evolution during a follow-up period that ranged from two to 277 months. Results: Five patients had anticardiolipin antibody titers over the cutoff for normal values, and two others were positive for anti- $\beta_{2}$-glycoprotein-I, without cross-reactivity. There was no difference in the incidence of thrombotic complications in the renal vein, or other locations, between these seven patients and the remaining patients. No differences in the clinical course of the nephropathy were detected either. Conclusions: Antiphospholipid antibodies may be found in patients with primary membranous nephropathy. They are not related to thrombosis or a worse evolution (Rev Méd Chile 2005; 133: 287-93).

(Key Words: Antibodies, anticardiolipin; Antibodies, antiphospholipid; Beta 2-glycoprotein I; Glomerulonephritis)

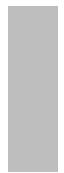

Recibido el 19 de octubre, 2004. Aceptado en versión corregida el 7 de enero, 2005.

Trabajo parcialmente financiado por Proyecto FONDECYT \# 1030263.

Laboratorio de Nefrología, Instituto de Medicina, Facultad de Medicina, Universidad Austral de Chile.

aTecnólogo Médico

L os anticuerpos antifosfolípidos asociados a trombosis en diferentes territorios, se han encontrado presentes en una amplia variedad de situaciones clínicas. Estos anticuerpos circulantes

Correspondencia a: Leopoldo G Ardiles. Laboratorio de Nefrología, Facultad de Medicina, Universidad Austral. Casilla 567, Valdivia, Chile. Tel./Fax +56 63215890.

E-mail: lardiles@uach.cl pueden ser detectados como anticoagulante lúpico en el plasma (a través de ensayos basados en las propiedades de inhibición de la coagulación in vitro) o como anticuerpos anticardiolipina (aCL) en el suero (utilizando ensayos de ELISA en fase sólida) ${ }^{1}$. Inicialmente descritos en pacientes con lupus, también se han encontrado acompañando a enfermedades glomerulares primarias ${ }^{2,3}$ y asociados a trombosis glomerular, tal como se ha encon- 
trado en la presentación catastrófica del síndrome antifosfolípido primario ${ }^{4}$. La nefropatía membranosa es la causa primaria más importante de síndrome nefrótico del adulto, dando cuenta de 30\% de las biopsias obtenidas en ese grupo. En adultos mayores, esta proporción llega hasta $50 \%$, siendo una entidad muy rara en la edad pediátrica $(<5 \%)$. Esta glomerulopatía es idiopática (o "primaria") en 85\% de los casos, pudiendo encontrarse asociada a carcinomas, lupus eritematoso sistémico (LES), uso de medicamentos, infecciones y trastornos metabólicos en una forma secundaria ${ }^{5}$. En su variedad primaria, afecta principalmente a sujetos de sexo masculino, y en algunos casos se ha descrito asociación con algunos antígenos HLA ${ }^{6}$. Su presentación clínica más común es el síndrome nefrótico puro, persistente en la mitad de los casos, pudiendo llegar a la insuficiencia renal crónica o muerte en $40 \%$ de los pacientes a 15 años de seguimiento. En su evolución natural, se manifiesta una alta frecuencia de complicaciones trombóticas, con una especial predisposición a localizarse en la vena renal, que sobrepasa lo observado en otras glomerulopatías que cursan con síndrome nefrótico ${ }^{7}$. Teniendo en cuenta que los anticuerpos anticardiolipina, cuando se asocian a complicaciones vasculares, están dirigidos a epítopes expresados en la $\aleph_{2}$-glicoproteína $1\left(\beta_{2} \mathrm{GP} 1\right)^{8-13}$, resolvimos conocer su incidencia, la reactividad cruzada con anti$\aleph_{2} \mathrm{GP1}$ y las posibles asociaciones con complicaciones trombóticas en la nefropatía membranosa idiopática.

\section{PACIENTES Y MÉTODOS}

Pacientes, muestras de suero y exámenes histológicos. Para estos estudios, se seleccionaron pacientes con el diagnóstico histológico inequívoco de nefropatía membranosa, estudiados entre los años 1985 y 1994, de los cuales se contara con muestras serológicas disponibles. Ellos fueron obtenidos del archivo sero-histológico existente en la Unidad de Nefrología de la Facultad de Medicina, Universidad Austral de Chile, Hospital Regional, Valdivia, el que funciona de acuerdo a un consentimiento informado aprobado por el comité de ética de la universidad. A través de la revisión de la ficha clínica y teniendo en cuenta los antecedentes clínicos y los exámenes de laboratorio, se pudo excluir a aquellos pacientes que, al momento de la toma de la muestra de sangre, recibían medicamentos o tenían alguna evidencia de infección. Así también se excluyó la posibilidad de LES y otras causas asociadas a la forma secundaria de la enfermedad.

Se puso especial atención en la presencia de complicaciones trombóticas. En el caso de la trombosis de la vena renal, ésta fue considerada cuando existía una confirmación angiográfica. Para las otras complicaciones potencialmente relacionadas con la presencia de antifosfolípidos, el diagnóstico fue considerado con el respaldo clínico, radiológico o de laboratorio apropiado. La evolución renal al final del seguimiento fue definida como remisión (ausencia de proteinuria con función renal normal), proteinuria (proteinuria en rangos no-nefróticos), nefrótico (proteinuria en rangos nefróticos) e insuficiencia renal crónica (creatinina estable sobre $2 \mathrm{mg} / \mathrm{dL}$, independiente del grado de proteinuria).

El tejido renal se obtuvo en todos los casos por biopsia renal percutánea, fijado en formalina al $4 \%$ e incluido en parafina para su examen en microscopia óptica con tinciones de hematoxilina-eosina, ácido periódico de Schiff (PAS) y metenamina de plata, poniendo especial atención a la presencia de trombosis glomerular y daño vascular oclusivo. Paralelamente, en tejido fresco congelado, se realizaron estudios de inmunofluorescencia indirecta para IgA, IgG, C3 y fibrina. Una porción del tejido previamente fijado fue examinado a través de microscopia electrónica tradicional, para efectuar la clasificación de acuerdo a lo recomendado por Ehrenreich y Churg, tal como hemos publicado previamente ${ }^{14}$. Las evaluaciones fueron realizadas en forma independiente por dos observadores, quienes lograron entera coincidencia en sus apreciaciones.

Anticardiolipina. Las muestras de suero, una vez obtenidas, fueron congeladas a $-70^{\circ} \mathrm{C}$ hasta efectuar los ensayos inmunológicos. Para identificar los anticuerpos anticardiolipina del isotipo IgG, se utilizó un ensayo inmunoenzimático en fase sólida (ELISA) que incluye la presencia de suero de bovino adulto, siguiendo las recomendaciones de Harris tal como se publicó previamente ${ }^{2,3}$. Brevemente, se cubrió placas de microELISA con $30 \mu \mathrm{L}$ 
de cardiolipina (SIGMA, C1649) a una concentración de $50 \mu \mathrm{g} / \mathrm{mL}$, diluida en etanol. Una mitad de la placa fue cubierta con etanol puro (pocillos blanco) para sustraer esa absorbancia del valor obtenido en los pocillos con antígeno y obtener así la absorción específica neta para cada muestra. Las placas fueron incubadas a $4^{\circ} \mathrm{C}$, durante la noche, para su evaporación y en la mañana siguiente fueron lavadas y bloqueadas con suero bovino adulto (ABS) al 10\% en buffer fosfato salino (PBS), incubando por $1 \mathrm{~h}$ a temperatura ambiente (TA). Las muestras de los pacientes fueron diluidas 1/50 en ABS al 10\% en PBS e incubadas a TA por $1 \mathrm{~h}$. Luego se lavó las placas por tres veces con PBS y se agregó un anticuerpo anti-IgG humana de origen caprino marcado con fosfatasa alcalina (SIGMA A3187) diluido en ABS al 10\% incubando a TA por 90 min. Se lavó las placas tres veces y se agregó el sustrato (p-nitrofenil-fosfato, $1 \mathrm{mg} / \mathrm{ml}$ ) para luego medir la absorbancia a $405 \mathrm{~nm}$. Las curvas de calibración se construyeron usando estándares purificados por afinidad con actividad conocida (Louisville APL
Diagnostics, Inc.) y los valores se expresan en GPLU. Los valores normales se establecieron estudiando el suero de 17 donantes normales del banco de sangre, definiendo como su límite superior a la suma del promedio +3 desviaciones estándar, considerando positivo a valores superiores a 7 GPLU en este estudio.

Anti- $\Omega_{2}$-glicoproteína 1. Los anticuerpos anti- $\Omega_{2}$ GP1 (IgG) fueron detectados utilizando un ELISA comercial (QUANTA Lite, INOVA Diagnostics Inc., San Diego, CA), siguiendo el procedimiento recomendado por el proveedor y expresando los resultados en unidades. Este ensayo considera como normales a valores bajo 20 unidades.

\section{RESULTADOS}

Veintiún pacientes fueron incluidos en este estudio (Tabla 1), siendo 5 del sexo femenino. El promedio de edad al momento de la biopsia renal fue de 40 años (rango 11-75). Dos pacientes

Tabla 1. H allazgos clínicos, serológicos y evolución de 21 pacientes con glomerulopatía membranosa idiopática

\begin{tabular}{|c|c|c|c|c|c|c|c|c|c|c|}
\hline $\begin{array}{c}\text { Caso } \\
\text { № }\end{array}$ & Género & $\begin{array}{l}\text { Edad } \\
\text { (años) }\end{array}$ & Estado ${ }^{\&}$ & $\begin{array}{l}\text { Tiempo toma } \\
\text { de muestra }\end{array}$ & $\mathrm{aCL}$ & $\begin{array}{l}\text { Anti- } \\
\beta_{2} \mathrm{GP1}\end{array}$ & TVR $^{*}$ & $\begin{array}{c}\text { Otras } \\
\text { complicaciones } \\
\text { específicas }^{@}\end{array}$ & Seguimiento\# & $\begin{array}{c}\text { Estado renal } \\
\text { al final } \\
\text { del seguimiento* }\end{array}$ \\
\hline 1 & $\mathrm{~F}$ & 53 & I & 21 & 3 & 2,4 & + & - & 24 & IRC \\
\hline 2 & M & 36 & III & 0 & 2 & 12,4 & + & Tromboembolismo crónico & 42 & Remisión \\
\hline 3 & $\mathrm{~F}$ & 15 & III & 0 & 10 & 1,87 & - & - & 72 & Remisión \\
\hline 4 & M & 42 & III & 0 & 1 & 3,6 & - & - & NO & $\mathrm{n} / \mathrm{d}$ \\
\hline 5 & M & 17 & I & 5 & 26 & 6,2 & + & Infarto cerebral frontal & 240 & Proteinuria \\
\hline 6 & M & 39 & II & 8 & 0 & 2,6 & . & - & 17 & Proteinuria \\
\hline 7 & $\mathrm{~F}$ & 45 & IV & 54 & 29 & 13,9 & - & - & 240 & Proteinuria \\
\hline 8 & M & 18 & II & 4 & 23 & 7,01 & - & - & 36 & IRC \\
\hline 9 & $\mathrm{M}$ & 41 & II & 0 & 4 & $\mathrm{NE}^{*}$ & - & - & 25 & IRC \\
\hline 10 & M & 34 & III & 0 & 1 & 1,8 & - & - & 3 & Nefrótico \\
\hline 11 & M & 28 & III & 0 & 1 & 1,9 & - & - & 2 & Nefrótico \\
\hline 12 & M & 75 & II & 0 & 3 & $\mathrm{NE}^{*}$ & - & - & 4 & Nefrótico \\
\hline 13 & $\mathrm{M}$ & 29 & IV & 0 & 3 & 2,9 & - & - & 10 & Nefrótico \\
\hline 14 & M & 50 & IV & 0 & 4 & 85,5 & - & - & 39 & Proteinuria \\
\hline 15 & M & 55 & II & 0 & 1 & 8,8 & + & Trombosis vena central retina & 168 & IRC \\
\hline 16 & $\mathrm{~F}$ & 11 & II & 64 & 6 & 2,6 & - & Pre-eclampsia & 228 & IRC \\
\hline 17 & M & 30 & II & 277 & 7 & 2,4 & - & Trombosis venosa profunda & 277 & IRC \\
\hline 18 & M & 23 & III & 158 & 4 & 2,1 & - & - & 216 & IRC \\
\hline 19 & $\mathrm{~F}$ & 63 & III & 0 & 53 & 2,6 & - & Trombocitopenia & 96 & IRC \\
\hline 20 & M & 73 & IV & 0 & 0 & 48,4 & - & - & 12 & Proteinuria \\
\hline 21 & M & 55 & III & 0 & 2 & 3,1 & - & Infartos cerebrales múltiples & 109 & IRC \\
\hline
\end{tabular}

\&: Etapa histológica. \#: Meses después de la biopsia. @: Potencialmente ligadas al síndrome antifosfolípido.

*: TVR= Trombosis de vena renal; NE= no estudiada; IRC= Insuficiencia Renal Crónica; $\mathrm{n} / \mathrm{d}=$ no disponible. 
tuvieron hallazgos histopatológicos correspondientes a la etapa I de Ehrenreich y Churg, 7 del estado II, 8 en estado III y 4 en estado IV. El seguimiento clínico estuvo disponible en 20 pacientes, alcanzando una mediana de 40 meses (rango 2-277 meses) posterior a la biopsia renal. Todos los pacientes se presentaron con síndrome nefrótico con la sola excepción del caso 17, que debutó con proteinuria en rangos no nefróticos. Nueve pacientes desarrollaron insuficiencia renal crónica, dos de ellos durante el primer año de observación y los otros entre 2 y 17 años después de la biopsia renal.

Cuatro pacientes desarrollaron trombosis de la vena renal: en 2 de ellos ocurrió al momento del diagnóstico (casos 2 y 5) y otros 2 (casos 1 y 15) veintitrés meses más tarde. Otras complicaciones, potencialmente relacionadas con la presencia de anticuerpos antifosfolípidos, se observaron en 7 pacientes y correspondieron a tromboembolismo pulmonar (caso 2), infarto cerebral frontal (caso 5), trombosis de vena central de la retina (caso 15), pre-eclampsia (caso 16), trombosis venosa profunda de extremidades inferiores (caso 17), trombocitopenia (caso 19) e infartos cerebrales múltiples (caso 21). En tres de los cuatro pacientes con trombosis de la vena renal se observó un segundo evento vascular durante el seguimiento (casos 2, 5 y 15). El análisis histopatológico de las biopsias renales no evidenció trombosis glomerular ni microvascular en ninguno de los casos.

Las muestras de suero fueron obtenidas al momento de la biopsia renal en 13 de los pacientes y en los restantes después del diagnóstico histológico. Anticuerpos anticardiolipina del isotipo IgG en títulos sobre el rango normal fueron detectados en un total de 5 pacientes, habiendo obtenido las muestras en dos de ellos al momento de la biopsia renal. Otros dos pacientes fueron positivos para anti- $\Omega_{2} \mathrm{GP1}$, ambas muestras tomadas al momento de la biopsia. No se observó reactividad cruzada para anticardiolipina y $\beta_{2} G P 1$ en ninguno de los sueros positivos. Un hecho interesante fue la aparición de un segundo evento trombótico extrarrenal en tres de los pacientes complicados de trombosis de vena renal, sin embargo solo uno de ellos fue positivo en anticardiolipina. Por otra parte, ninguno de los pacientes afectados de trombosis de la vena renal así como ninguno de los que desarrollaron complicaciones potencialmente relacionadas con el síndrome antifosfolípido presentó positividad para anti- $\beta_{2}$ GP1. Finalmente, la positividad para anticardiolipina o $\beta_{2} \mathrm{GP1}$ no se asoció con una mayor predisposición al desarrollo de insuficiencia renal crónica durante el seguimiento.

\section{DisCUSIÓN}

Cinco de los 24 pacientes (20\%) con nefropatía membranosa idiopática mostrados en este estudio presentaron anticuerpos anticardiolipina, sin demostrarse reactividad cruzada contra $\aleph_{2}$-GP1 ni una particular predisposición al desarrollo de trombosis.

La detección de anticuerpos antifosfolípidos del tipo anticardiolipina en pacientes con glomerulopatías ${ }^{15}$, particularmente en aquellos con trastornos inmunológicos subyacentes como el LES, ha sido reportada con incidencia variable ${ }^{16}$. Estos anticuerpos pueden ser detectados como anticoagulante lúpico o anticuerpos anticardiolipina ${ }^{1}$ y datos publicados durante la última década, han demostrado que reconocen a proteínas plasmáticas afines a fosfolípidos como sus blancos antigénicos. Los mecanismos in vivo propuestos para el rol trombogénico de los anticuerpos antifosfolípidos, incluyen la interferencia con la cascada de la coagulación, llevando a un estado procoagulante a través de la inhibición de las vías de la proteína $\mathrm{C}$ activada y la antitrombina III, una inhibición de la fibrinolisis y aumento de la actividad del factor tisular. Otras proteínas involucradas en la coagulación tales como protrombina, proteínas $\mathrm{C}, \mathrm{S}$ y anexina $\mathrm{V}$ pueden ser también blanco de ellos 1,17 .

Datos publicados durante la última década han mostrado que los anticuerpos anticardiolipina, altamente purificados, no se unen al antígeno anticardiolipina en placas de ELISA, a menos que los agentes bloqueadores contengan suero de bovino o humano, ocurriendo un fenómeno similar al intentar su unión específica a columnas. Esto sugiere que la interacción requiere de la presencia de un cofactor presente en la sangre, actualmente identificado como la $\beta_{2} \mathrm{GP} 1^{18-20}$. Coincidente con ello, se ha demostrado que los anticuerpos anti- 
cardiolipina asociados a trombosis están dirigidos principalmente contra epítopes expresados en la $\aleph_{2} \mathrm{GP1}$, pero no en cardiolipina ${ }^{8-13}$. Además, como $\beta_{2} \mathrm{GP1}$ puede actuar como un anticoagulante in vivo, los anticuerpos dirigidos contra esta molécula pueden interferir con ese rol ${ }^{19,21}$.

El uso de ensayos con anti- $\Omega_{2} \mathrm{GP} 1$ en el estudio de las complicaciones trombóticas del síndrome antifosfolípido han mostrado una mayor especificidad diagnóstica, porque éstos no detectarían los anticuerpos independientes de $\aleph_{2} \mathrm{GP} 1$, que no estarían asociados con las complicaciones ${ }^{22-24}$. Es así como ocurre en los estados infecciosos en que pueden aparecer anticuerpos anticardiolipina sin esta reactividad cruzada ni complicaciones trombóticas ${ }^{15,25}$. Nosotros preferimos excluir en nuestra selección a aquellos pacientes que tuvieran algún elemento de sospecha de infección activa para evitar esta variable.

La coagulación intravascular y los episodios tromboembólicos son reconocidas complicaciones del síndrome nefrótico, siendo muy característico el compromiso oclusivo de la vena renal, particularmente en presencia de glomerulopatía membranosa ${ }^{26}$. Aunque múltiples factores han sido propuestos como causales de esta predisposición, la asociación predominante entre trombosis venosa renal y glomerulopatía membranosa permanecen sin una explicación satisfactoria ${ }^{27,28}$. Aunque algunos estudios han planteado una relación con la presencia de anticuerpos antifosfolípidos en la nefropatía membranosa lúpica ${ }^{29}$, no se ha planteado algo similar para la variedad idiopática de esta glomerulopatía. En nuestro análisis, no observamos una mayor incidencia de anticuerpos anticardiolipina en los pacientes que se complicaron de trombosis de la vena renal. Merece ser destacado que, 3 de estos cuatro pacientes (casos 2, $5 \mathrm{y}$ 15) desarrollaron otros accidentes trombóticos, demostrando una particular predisposición a estas complicaciones, pero dado que solo uno de ellos mostró positividad para anticardiolipina y sin reactividad coincidente para $\beta_{2} \mathrm{GP1}$, no podemos asumir alguna relación patogénica con los anticuerpos antifosfolípidos.

Los títulos de aCL demostrados en nuestros pacientes fueron esencialmente bajos y actualmente se acepta que el riesgo de trombosis se asocia a una mayor tasa de anticuerpos ${ }^{30-32}$. Esto, sumado a la falta de reactividad cruzada para $\aleph_{2} \mathrm{GP1}$, podría explicar la ausencia de complicaciones vasculares. Desafortunadamente, los dos pacientes anti- $\beta_{2} \mathrm{GP1}$ positivos no tuvieron un seguimiento muy largo y no dispusimos de muestras seriadas para confirmar la persistencia de nuestros hallazgos, ni la aparición de eventos trombóticos en el largo plazo. Merece ser mencionada la existencia de publicaciones aludiendo a una baja transitoria de los títulos de antifosfolípidos durante la evolución de los fenómenos trombóticos $^{33-35}$. En nuestra serie, solo en el caso 2 existió una coincidencia entre la presencia de trombosis activa de vena renal. En el caso 1, la muestra fue tomada 1 mes previo a la trombosis y en todos los otros casos no hubo relación temporal con el episodio vascular u otro evento potencialmente ligado al síndrome antifosfolípido, por lo que no debiéramos esperar una gran probabilidad de falsos negativos.

La presencia de anticuerpos anticardiolipina en el lupus ha resultado ser un poderoso predictor de trombosis intraglomerular y su hallazgo, tanto como la presencia de aCL, indican un peor pronóstico renal a largo plazo ${ }^{36}$. La evaluación de las biopsias renales en nuestra serie no demostró presencia de trombos intraglomerulares y el seguimiento clínico tampoco mostró una peor evolución de la función renal en los pacientes aCL positivos.

Aunque aquellos pacientes que se complicaron de trombosis no eran precisamente los que presentaban un síndrome nefrótico más grave, en términos de proteinuria e hipoalbuminemia, es razonable suponer que otros factores asociados con el síndrome nefrótico mismo pueden haber sido más importantes en la patogénesis de las complicaciones observadas. Con nuestros datos no podemos descartar que la presencia de estos anticuerpos constituya solo un epifenómeno, parte de una respuesta inmune alterada, en una enfermedad inmunológicamente mediada. Estudios prolongados y prospectivos con mayor número de casos pueden ayudar a descartar definitivamente un rol patogénico de estos anticuerpos en esta enfermedad.

Finalmente, un asunto que merece un comentario adicional, es la detección de sero-reactividad de especificidad inesperada durante la evaluación 
de una glomerulopatía primaria. La presencia de marcadores inmunológicos sin rol patogénico ni significado pronóstico, puede ser el resultado de una activación policlonal de los linfocitos $\mathrm{B}$ en los desórdenes inmunológicos ${ }^{37}$. Muchos de nuestros pacientes, seguidos por largos períodos, no evidenciaron criterios diagnósticos de lupus, neoplasias ni otras enfermedades asociadas con la variedad secundaria de glomerulopatía membranosa. La evolución clínica y la ausencia de

\section{REFERENCIAS}

1. HANLY JG. Antiphospholipid syndrome: an overview. CMAJ 2003; 168: 1675-82.

2. Ardiles L, Ramírez P, Moya P, Caorsi I, Mezzano S. Anticardiolipin antibodies in acute poststreptococcal glomerulonephritis and streptococcal impetigo. Nephron 1999; 83: 47-52.

3. Ardiles LG, Olavarría F, Elgueta M, Moya P, Mezzano S. Anticardiolipin antibodies in classic pediatric hemolytic-uremic syndrome: a possible pathogenic role. Nephron 1998; 78: 278-83.

4. Amigo MC, García T, Robles M, Bochicchio T, Reyes PA. Renal involvement in primary antiphospholipid syndrome. J Rheumatol 1992; 19: 1181-5.

5. Falk RJ, Jennette JC, Nachman PH. Primary Glomerular Disease. In Brenner \& Rector's The Kidney, ed Brenner BM, WB Saunders, 2000; 1284-92.

6. Mezzano S, Rojas G, Ardiles L, Caorsi I, Bertoglio JC, LÓPEZ MI ET AL. Idiopathic membranous nephropathy, associated with HLA-DRw3 and not related to monocyte-phagocyte system Fc receptor dysfunction, in father and son. Nephron 1991; 58: 320-4.

7. Couser WG, SHankiand SJ. Membranous Nephropathy. In: Comprehensive Clinical Nephrology, ed Johnson RJ \& Feehally J, Elsevier, 2003; 295307.

8. Matsuura E, Igarashi Y, Yasuda T, Triplett DA, KoIKE T. Anticardiolipin antibodies recognize beta 2-glycoprotein I structure altered by interacting with an oxygen modified solid phase surface. J Exp Med 1994; 179: 457-62. marcadores serológicos, respaldan la naturaleza primaria o idiopática de la patología presentada en esta comunicación. Los anticuerpos anticardiolipina, tal como ocurre en otras enfermedades glomerulares ${ }^{15}$, pueden estar presentes en el suero de pacientes con nefropatía membranosa, sin estar asociados a trombosis. Finalmente, y algo que puede ser muy importante, su detección no apunta necesariamente a una etiología secundaria, lúpica, de la enfermedad.

9. Hunt J, KRILS S. The fifth domain of beta 2glycoprotein I contains a phospholipid binding site (Cys281-Cys288) and a region recognized by anticardiolipin antibodies. J Immunol 1994; 152: 653-9.

10. Roubey RA, EIsenberg RA, HaRper MF, Winfield JB. "Anticardiolipin" autoantibodies recognize beta 2glycoprotein I in the absence of phospholipid. Importance of Ag density and bivalent binding. J Immunol 1995; 154: 954-60.

11. Pengo V, Biasiolo A, Fior MG. Autoimmune antiphospholipid antibodies are directed against a cryptic epitope expressed when beta 2-glycoprotein I is bound to a suitable surface. Thromb Haemost 1995; 73: 29-34.

12. Arvieux J, Roussel B, Jacob MC, Colomb MG. Measurement of anti-phospholipid antibodies by ELISA using beta 2-glycoprotein I as an antigen. J Immunol Methods 1991; 143: 223-9.

13. VIARD JP, AMOURA Z, BACH JF. Association of anti-beta 2 glycoprotein I antibodies with lupus-type circulating anticoagulant and thrombosis in systemic lupus erythematosus. Am J Med 1992; 93: 181-6.

14. Ardiles L, Mezzano S, Arriagada A, Caorsi I. Clinical course in 51 patients with idiopathic membranous glomerulopathy. Rev Méd Chile 1989; 117: 266-72.

15. Quereda C, Otero GG, Pardo A, Orte L, Rivera M, GonZALO A ET AL. Prevalence of antiphospholipid antibodies in nephropathies not due to systemic lupus erythematosus. Am J Kidney Dis 1994; 23: $555-61$.

16. Frampton G, Hicks J, Cameron JS. Significance of anti-phospholipid antibodies in patients with lupus nephritis. Kidney Int 1991; 39: 1225-31. 
17. LeVine JS, Branch DW, Rauch J. The antiphospholipid syndrome. N Engl J Med 2002; 346: 752-63.

18. Gaw M, Comfurius P, Maassen C, Hemker HC, De BaEts MH, Van BReda-VRIESMan PJ V et al. Anticardiolipin antibodies (ACA) directed not to cardiolipin but to a plasma protein cofactor. Lancet 1990; 335:1544-7.

19. Mcneil HP, Simpson RJ, Chesterman CN, Krils SA. Anti-phospholipid antibodies are directed against a complex antigen that includes a lipid-binding inhibitor of coagulation: beta 2-glycoprotein I (apolipoprotein H). Proc Natl Acad Sci USA 1990; 87: 4120-4.

20. Matsuura E, Igarashi Y, Fujimoto M, Ichikawa K, KoIKe T. Anticardiolipin cofactor(s) and differential diagnosis of autoimmune disease. Lancet 1990; 336: 177-8.

21. Kandiah DA, KriLs SA. Beta 2-glycoprotein I. Lupus 1994; 3: 207-12.

22. Roubey RA, MaLdonado MA, Byrd SN. Comparison of an enzyme-linked immunosorbent assay for antibodies to beta 2-glycoprotein I and a conventional anticardiolipin immunoassay. Arthritis Rheum 1996; 39: 1606-7.

23. Cabiedes J, Cabral aR, Alarcón S. Clinical manifestations of the antiphospholipid syndrome in patients with systemic lupus erythematosus associate more strongly with anti-beta 2-glycoproteinI than with antiphospholipid antibodies. J Rheumatol 1995; 22: 1899-906.

24. Balestrieri G, Tincani A, Spatola L, Auegri F, Prati E, Cattaneo R et al. Anti-beta 2-glycoprotein I antibodies: a marker of antiphospholipid syndrome? Lupus 1995; 4: 122-30.

25. McNeil HP, CheSTeRman CN, KriLs SA. Immunology and clinical importance of antiphospholipid antibodies. Adv Immunol 1991; 49: 193-280.

26. Liach F, Koffler A, Finck E, Massry SG. On the incidence of renal vein thrombosis in the nephrotic syndrome. Arch Intern Med 1977; 137: 333-6.

27. LAсн F. Hypercoagulability, renal vein thrombosis, and other thrombotic complications of nephrotic syndrome. Kidney Int 1985; 28: 429-39.
28. HaRRIS RC, Ismail N. Extrarenal complications of the nephrotic syndrome. Am J Kidney Dis 1994; 23: 477-97.

29. Zea M, Rodríguez G, Irigoyen O, Vázquez D, Pardo V, Mampaso FM et al. Antiphospholipid antibodies in systemic lupus erythematosus: incidence, significance and relation to lupus nephritis. Med Clin (Barc) 1989; 92: 724-8.

30. Alarcón S, Deleze M, Oria CV, Sánchez G, Gómez P, CABIEDES J ET AL. Antiphospholipid antibodies and the antiphospholipid syndrome in systemic lupus erythematosus. A prospective analysis of 500 consecutive patients. Medicine (Baltimore) 1989; 68: 353-65.

31. Levine SR, Salowich P, Sawaya KL, Perry M, Spencer HJ, WinKLER HJ ET AL. IgG anticardiolipin antibody titer $>40 \mathrm{GPL}$ and the risk of subsequent thrombo-occlusive events and death. A prospective cohort study. Stroke 1997; 28: 1660-5.

32. Sairam S, Baethge BA, McNearney T. Analysis of risk factors and comorbid diseases in the development of thrombosis in patients with anticardiolipin antibodies. Clin Rheumatol 2003; 22: 24-9.

33. Divate S, Hardikar P, Bichile LS, Rajadhyaksha A. Transient low levels of antibodies at initial presentation of primary anti-phospholipid syndrome. J Assoc Physicians India 2003; 51: 722-3.

34. Drenkard C, SÁnchez-Guerrero J, Arcon-Segovia D. Fall in antiphospholipid antibody at time of thromboocclusive episodes in systemic lupus erythematosus. J Rheumatol 1989; 16: 614-7.

35. Miret C, Cervera R, Reverter JC, García-Carrasco M, Ramos M, Moша M et al. Antiphospholipid syndrome without antiphospholipid antibodies at the time of the thrombotic event: transient 'seronegative' antiphospholipid syndrome? Clin Exp Rheumatol 1997; 15: 541-4.

36. Bhandari S, Harnden P, Brownohn AM, Turney JH. Association of anticardiolipin antibodies with intraglomerular thrombi and renal dysfunction in lupus nephritis. QJM 1998; 91: 401-9.

37. EGNER W. The use of laboratory tests in the diagnosis of SLE. J Clin Pathol 2000; 53: 424-32. 